\title{
PERCEPÇÃO SOBRE A ALTA HOSPITALAR DE PESSOAS INTERNADAS EM UNIDADE DE ATENÇÃO PSICOSSOCIAL
}

\section{People's perception of hospital discharge from a psychosocial care center
Percepción de personas ingresadas en unidad de atención psicosocial sobre la alta hospitalaria

\author{
Valentine Cogo Mendes (iD \\ Universidade Federal de Santa Maria (UFSM) - Santa Maria - RS - Brasil

\section{Zaira Letícia Tisott iD} \\ Universidade Federal do Rio Grande do Sul (UFRGS) \\ Valquíria Toledo Souto iD \\ Universidade Federal de Santa Maria (UFSM) - Santa Maria - RS - Brasil \\ Marlene Gomes Terra (iD \\ Universidade Federal de Santa Maria (UFSM) - Santa Maria - RS - Brasil \\ Daiana Foggiato Siqueira (iD \\ Universidade Federal de Santa Maria (UFSM) - Santa Maria - RS - Brasil \\ Bruno Vinicius Rodrigues iD \\ Universidade Federal de Santa Maria (UFSM) - Santa Maria - RS - Brasil
}

\section{RESUMO}

Objetivo: Compreender a percepção de pessoas em sofrimento psíquico internadas em uma unidade de atenção psicossocial sobre a alta hospitalar. Métodos: Estudo qualitativo desenvolvido com 13 pessoas em sofrimento psíquico internadas em uma Unidade de Atenção Psicossocial de um hospital de ensino do estado do Rio Grande do Sul, Brasil. A coleta dos dados foi realizada por meio da entrevista semidirigida, realizada em outubro de 2016, e posteriormente analisada pela estratégia de análise de conteúdo. Emergiram duas categorias: Significados atribuídos à alta hospitalar; Estratégias para continuidade do tratamento diante da alta hospitalar. Resultados: Foi possível perceber alguns significados acerca da alta hospitalar para os usuários internados, como sentimentos de medo, angústia, recomeço e liberdade, além de representar uma estratégia para a continuidade do cuidado. Conclusão: A alta hospitalar é compreendida de diversas maneiras, despertando sentimentos, expectativas e desejos para a pessoa em sofrimento psíquico que está saindo de uma unidade de internação.

Descritores: Saúde Mental; Transtornos Mentais; Alta do Paciente; Enfermagem.

\section{ABSTRACT}

Objective: To understand the perception people experiencing psychic suffering and hospitalized in a psychosocial care center have about hospital discharge. Methods: This qualitative study was conducted with 13 people experiencing psychic suffering hospitalized in a Psychosocial Care Center of a teaching hospital in the state of Rio Grande do Sul, Brazil. Data were collected using semi-directed interviews held in October 2016 and then analyzed using the content analysis strategy. A total of two categories emerged: meanings attributed to hospital discharge; Strategies for continuity of treatment in the face of hospital discharge. Results: It was possible to notice some meanings attributed to hospital discharge by hospitalized users, such as feelings of fear, anguish, starting over, and freedom. In addition, it represents a strategy for continuity of care. Conclusion: Hospital discharge is understood in several ways and it arouses feelings, expectations and desires in people experiencing psychic suffering who are leaving an inpatient center.

Descriptors: Mental Health; Mental Disorders; Patient Discharge; Nursing.

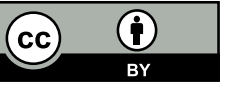




\section{RESUMEN}

Objetivo: Comprender la percepción de personas con sufrimiento psíquico ingresadas en una unidad de atención psicosocial sobre la alta hospitalaria. Métodos: Estudio cualitativo desarrollado con 13 personas con sufrimiento psíquico ingresadas en una Unidad de Atención Psicosocial de un hospital de enseñanza del estado de Rio Grande do Sul, Brasil. La recogida de datos se dio a través de entrevista semidirigida realizada en octubre de 2016 y ha sido analizada por el análisis de contenido. Dos categorías han emergido: Significados de la alta hospitalaria; Estrategias para el seguimiento del tratamiento ante la alta hospitalaria. Resultados: Se ha percibido algunos significados acerca de la alta hospitalaria para los usuarios ingresados como los sentimientos de miedo, la angustia, el recomienzo y la libertad, además de representar una estrategia para el seguimiento del cuidado. Conclusión: Se comprende la alta hospitalaria de diversas maneras, despertando los sentimientos, las expectativas y los deseos para la persona con sufrimiento psíquico que deja la unidad de internación.

Descriptores: Salud Mental; Trastornos Mentales; Alta del Paciente; Enfermería.

\section{INTRODUÇÃO}

Historicamente, as pessoas consideradas "loucas" eram retiradas do convívio social e institucionalizadas nos chamados manicômios, onde ficavam até o fim de suas vidas, sem contato com o mundo. Pessoas avaliadas "loucas", ou que não possuíssem condições sociais, e que vagavam pelas ruas, eram aprisionadas para manter a ordem social ${ }^{(1)}$.

Contudo, depois de vários movimentos e lutas sociais, tanto da sociedade organizada como de profissionais de saúde; ocorreram mudanças nos métodos de cuidado no campo da saúde mental. Como marco referencial, a Lei n. ${ }^{\circ} 10.216$, conhecida como Lei da Reforma Psiquiátrica, que avança em direção ao atendimento das pessoas em sofrimento psíquico visando a sua desinstitucionalização, reinserindo-as na sociedade com o apoio dos serviços substitutivos. A Reforma Psiquiátrica brasileira prevê a desconstrução do modelo hospitalocêntrico e manicomial, buscando transformações no cuidado às pessoas com transtorno mental, de forma que o tratamento ocorra, preferencialmente, em seu território. $O$ seu objetivo é repensar a forma de agir e cuidar das pessoas em sofrimento psíquico(2).

Nesse contexto, a internação psiquiátrica deve ser um recurso terapêutico utilizado quando forem esgotadas todas as outras possibilidades pautadas nos cuidados em rede de atenção à saúde ${ }^{(3)}$. Assim, a internação se faz necessária quando a pessoa apresenta-se em situação de grave sofrimento psíquico, que pode ser caracterizada por momentos de crise, agressividade, situações em que o usuário coloque a si ou a outras pessoas em risco(4), situações que não possam ser resolvidas na atenção primária ou em serviço especializado.

As ações oferecidas durante a internação devem ser baseadas no cuidado humanizado e sensível, estimulando o autocuidado, o vínculo familiar e social, visando fortalecer as relações. Além disso, é preciso instigar a corresponsabilização no tratamento, pautado na autonomia nas atividades cotidianas para oportunizar a capacidade do indivíduo em gerar sentido para as coisas, seu valor social e, assim, restabelecer seu papel enquanto cidadão(5)

A duração de uma internação varia de acordo com a situação psiquiátrica, clínica e social de cada pessoa. Por vezes, a internação por um longo período de tempo gera desconforto e anseio para voltar para casa. Desse modo, o planejamento da alta hospitalar é uma ferramenta indispensável para o cuidado integral, pois se busca o autocuidado continuado da pessoa em seu domicílio( ${ }^{(6)}$.

A Política Nacional de Promoção da Saúde sinaliza a importância de se investir em formas mais amplas de intervir em saúde, de organizar uma rede de atenção a essas pessoas, convocando todos os recursos afetivos, sanitários, sociais, econômicos, culturais e de lazer para a produção do cuidado integral(7) e, assim, levar o indivíduo à promoção da sua saúde.

A realização do estudo aqui proposto justifica-se pela necessidade de dar voz às pessoas que vivenciaram um período de internação hospitalar em unidade de atenção psicossocial, para que possam relatar sua visão acerca dessa experiência e seus determinantes. Diante do exposto, o estudo teve como questão de pesquisa: como a pessoa em sofrimento psíquico internada em uma unidade de atenção psicossocial percebe a alta hospitalar?

Dessa forma, objetivou-se compreender a percepção de pessoas em sofrimento psíquico internadas em uma unidade de atenção psicossocial sobre a alta hospitalar. 


\section{MÉTODOS}

Trata-se de uma pesquisa qualitativa ${ }^{(8,9)}$, da qual participaram pessoas em sofrimento psíquico que estavam internadas na Unidade de Atenção Psicossocial de um hospital de ensino de um município do Rio Grande do Sul, Brasil. A referida unidade dispõe, atualmente, de 30 leitos para internação de adultos, de ambos os sexos, em situação de sofrimento psíquico grave. $O$ tempo de internação varia de 15 a 45 dias, de acordo com a situação de cada usuário.

$\mathrm{Na}$ escolha dos entrevistados, foram selecionados pacientes de ambos os sexos, internados há pelo menos sete dias e que apresentassem condições cognitivas para compreender os objetivos da pesquisa. Não integraram o estudo as pessoas que não estavam em condições de verbalizar seus sentimentos. Essa seleção foi possível com a colaboração dos trabalhadores do serviço, que indicaram, entre aqueles que estavam internados no período da coleta de dados, os potenciais participantes. Assim, chegou-se a 13 participantes por exaustão de dados, que ocorre quando são incluídos na pesquisa todos os participantes disponíveis diante dos critérios ${ }^{(10)}$

A coleta de dados ocorreu no mês de outubro de 2016, por meio da realização de entrevistas individuais semidirigidas. Essa técnica de coleta de dados foi escolhida a fim de que a entrevistadora pudesse disparar as questões previamente definidas, porém com flexibilidade, para que o entrevistado também assumisse o comando em alguns momentos ${ }^{(9)}$. As entrevistas foram guiadas por um instrumento contendo questões fechadas acerca das variáveis: sexo, idade, tempo de internação, estado civil e uma questão norteadora: "Fale-me, o que você sabe acerca da sua alta hospitalar?".

As entrevistas duraram cerca de 30 minutos, sendo realizadas em uma sala reservada na unidade de internação, observando o cuidado para que oferecesse um ambiente livre de interferência externa e com garantia da privacidade e sigilo dos participantes. Todos os participantes foram esclarecidos sobre os objetivos da pesquisa e os seus aspectos éticos relacionados, tendo a coleta de dados iniciado somente após autorização por escrito mediante assinatura do Termo de Consentimento Livre e Esclarecido (TCLE) em duas vias. As entrevistas foram gravadas com gravador e transcritas para posterior análise.

A análise dos dados ocorreu de acordo com a técnica de análise de conteúdo ${ }^{(9)}$. A primeira etapa consistiu na pré-análise, etapa em que se realizaram leituras flutuantes para estabelecer contato inicial com o texto transcrito. Ocorreram leituras e releituras até que as pesquisadoras pudessem impregnar-se pelo conteúdo. A segunda etapa, de categorização, ocorreu pela classificação de fragmentos do texto segundo critérios de relevância e repetição, e transformou os dados brutos em organizados. A terceira etapa consistiu na apresentação dos resultados de forma descritiva e na discussão com a literatura e inferências ${ }^{(9)}$. Emergiram duas categorias: Significados atribuídos à alta hospitalar; Estratégias para continuidade do tratamento diante da alta hospitalar.

Para preservar a identidade, privacidade e o sigilo, neste manuscrito os depoimentos dos participantes são referenciados utilizando-se a letra "P", inicial da palavra participante, seguida de um algarismo arábico (P1, P2, ...). Em todas as suas etapas este estudo seguiu os princípios e diretrizes da Resolução $n .^{\circ} 466 / 12^{(11)}$ do Conselho Nacional de Saúde, sendo aprovado pelo Comitê de Ética em Pesquisa (CEP) da instituição de origem com o Registro n. ${ }^{\circ} 1.740 .283$.

\section{RESULTADOS E DISCUSSÃO}

Neste espaço serão expostos os dados de identificação dos entrevistados. A seguir serão apresentadas e discutidas as categorias que foram elucidadas do estudo: Significados atribuídos à alta hospitalar; Estratégias para continuidade do tratamento diante da alta hospitalar.

\section{Dados de identificação dos participantes}

Das 13 pessoas entrevistadas, 11 eram mulheres e dois, homens, com idades de 30 a 54 anos. Em relação ao estado civil, sete participantes eram casados, e os outros seis eram solteiros. O tempo de internação dessas pessoas variou entre 15 dias a dois meses. Quanto ao número de internações, a maioria possuía histórico de reinternações.

\section{Significados atribuídos à alta hospitalar}

Nessa categoria descreve-se a percepção dos participantes sobre a alta e o seu significado para o tratamento. A alta é percebida pelos entrevistados como um momento de vitória, de liberdade e recomeço, não apenas no tratamento, mas na vida: 
"A alta, para mim, representa uma vitória, um sinal que o doutor acha que eu já tenho capacidade de enfrentar a vida." (P2)

"[...] Uma nova perspectiva de vida, a gente tem que estar bem estabilizado aqui para sair." (P6)

"A alta significa uma nova vida, um novo recomeço, e o passado deixado para trás e sair curada daqui de dentro." (P10)

"A alta significa que eu vou ficar livre em casa, ajudando a mãe. Livre para estar em casa, poder dar uma saída." (P11)

A percepção da alta atrelada à sensação de liberdade hospitalar ainda "aprisiona" para estabilizar. A internação, apesar das mudanças no tratamento advindas da Reforma Psiquiátrica, sinaliza que o tratamento utiliza regras de funcionamento e convívio limitadoras, ainda exige que a pessoa se adapte a um local para sua reabilitação psicossocial, o que pode suscitar sentimentos de aprisionamento na pessoa em tratamento ${ }^{(12)}$. Por isso, ao sair do hospital, a pessoa vivencia um sentimento de liberdade, de poder voltar a realizar atividades de acordo com seus desejos.

Essa percepção, que relaciona a internação à prisão e a alta à liberdade, soma-se ao evidenciado em outro estudo com pessoas internadas em unidade psiquiátrica, que sinaliza que, diante de um quadro de melhora e possibilidade de alta, há no paciente a ideia de cura e a mascarada possibilidade de liberdade, por acreditar que não necessita mais de tratamento ${ }^{(13)}$.

A alta hospitalar, vista como recomeço, remete à ideia de reiniciar a vida, esquecer acontecimentos do passado, o que aparece em uma das falas ainda atrelado à ideia de cura, de que a internação e os ajustes das medicações possam deixar a pessoa curada. Além disso, é necessário reforçar junto aos usuários o papel dos serviços extra-hospitalares, como unidades básicas de saúde, CAPS, entre outros, para a continuidade do tratamento. Ademais, os hospitais gerais que possuem unidade de saúde mental devem prestar o cuidado pautado na humanização, colocando o usuário como protagonista do seu cuidado, e não o relegando a cuidados somente tecnicistas e com o mínimo de sensibilidade.

Há, em contrapartida, uma pessoa que percebe a alta como um acontecimento simples, sem tantas expectativas:

"Nada demais, porque eu acho eu estou tão bem assim, tão lúcida. Na minha lucidez normal que eu acho que já está na hora de eu voltar pra casa [...]. Mas, na expectativa, é isso aí, que eu estou bem. Não é aquela expectativa: quero ir embora, quero ir embora. Aquele desespero que dá em todo mundo." (P9)

A participante refere que a alta não é nada demais. Percebe-se que a pessoa em sofrimento psíquico, por estar estabilizada, sente que a alta é um acontecimento natural para quem passa por algum período internado em alguma unidade hospitalar. E que, apesar de estar bem e poder ir embora, não desencadeia angústia. Sentir-se bem pode ser o sinônimo de ter confiança no tratamento que foi oferecido, a garantia de que já está estabilizada, o que gera segurança, e a ideia de que esteja bem para enfrentar a situação da melhor maneira. No entanto também pode significar que, após receber a alta hospitalar, o usuário, por sentir-se bem e estabilizado, não siga o tratamento terapêutico indicado, o que pode originar um novo quadro de hospitalização(13).

O período de internação em uma unidade de atenção psicossocial provoca uma mistura de sentimentos na pessoa que sofre com algum transtorno psíquico. Observa-se nas falas a seguir também o sentimento de medo da reincidência de uma crise, de insegurança diante da alta:

"Eu penso que eu tenho medo de voltar para casa, da alta; ficar bem e, daqui uns dias, voltar a coisa toda de novo, como era antes." (P1)

"Eu estou me sentindo muito bem agora, mas eu tenho medo que volte a acontecer isso. Eu não vou com toda a força, porque eu tenho pé atrás, porque já deu recaída uma vez." (P5)

Esses sentimentos trazem a compreensão de que a internação é um acontecimento que proporciona relativa segurança, por saber que na Unidade de Atenção Psicossocial haverá a organização necessária para o restabelecimento de sua saúde mental: alimentação e medicações em horário regular, sono satisfatório, atendimento psiquiátrico e psicológico, e grupos terapêuticos, entre outras atividades constantes. No retorno ao lar, a pessoa poderá sentir-se desamparada e ao deparar-se com a realidade do meio em que vive, pode sofrer confronto, podendo desencadear uma nova crise ${ }^{(14)}$.

Um estudo que acompanhou e avaliou a adesão da pessoa em sofrimento psíquico aos tratamentos após sua alta hospitalar demonstrou que os usuários não possuíam proposta terapêutica para o pós-alta e que o atendimento das 
equipes não está centrado na reabilitação psicossocial ${ }^{(15)}$. Esse resultado pode indicar a fragilidade do planejamento da alta hospitalar junto ao usuário e família, que podem ficar desamparados.

Além disso, o ambiente familiar que a pessoa em sofrimento psíquico está inserida pode interferir na adesão ao tratamento e na diminuição dos sentimentos de ansiedade e medo no momento da alta. As relações familiares são mediadas cotidianamente por emoções decorrentes das dificuldades impostas pelo sofrimento psíquico. Desse modo, é essencial que a família esteja ciente acerca das condições do sofrimento e, assim, forme, juntamente com a pessoa, uma rede de apoio para que exista sentimento de segurança no retorno ao $\operatorname{lar}^{(15)}$.

A alta hospitalar também significou a possibilidade de retornar ao ambiente familiar:

"Ah! Eu vejo como retorno ao lar. Retorno à minha casinha, ao meu marido, ao meu filho, meu cachorrinho." (P2)

"[...] a alta sempre é bem-vinda. A gente volta para familia, para a sociedade." (P4)

"É voltar para casa, voltar pros amores da gente. Voltar pra casa." (P7)

"A alta, ah, tudo de bom! A minha pessoa quer ir para casa para ver meus filhos, para ver meus netos, eu tenho vontade." (P12)

Se antigamente as pessoas com transtornos mentais eram institucionalizadas e afastadas do lar de forma definitiva, as mudanças na lógica do cuidado em saúde mental deram início a um importante debate sobre a inclusão da família no tratamento, a qual pode ser compreendida como uma oportunidade para acolher e cuidar das relações entre os seus membros. Esse processo, por vezes, é descrito como doloroso e difícil, mas impacta positivamente para as interações familiares no decorrer do tratamento ${ }^{(16)}$.

A família possui influência direta na recuperação das pessoas em sofrimento psíquico. No entanto, no cotidiano dos serviços, pode ocorrer que o enrijecimento de algumas rotinas acaba ainda restringindo a presença da família fora de horários de visita pré-determinados, constituindo-se em entraves significativos para a posterior reinserção dos pacientes no convívio familiar ${ }^{(17)}$.

Uma estratégia importante de inclusão e cuidado aos familiares de pessoas com transtornos mentais durante a internação e o preparo para a alta hospitalar é a realização de intervenções em grupo. O grupo de familiares tem sido visto como um espaço potente para o cuidado das famílias, repercutindo positivamente no tratamento ${ }^{(18)}$. Aquelas que participam desse tipo de atividade a descrevem como um espaço de apoio mútuo e de fortalecimento, no qual se compartilha informações sobre a doença e discutem-se conjuntamente as dúvidas e dificuldades sobre o tratamento ${ }^{(18)}$.

As percepções que relacionam a alta ao retorno ao ambiente familiar refletem a perspectiva de pessoas que ainda possuem o lar como uma possibilidade, porém deve-se também considerar que há pessoas internadas que não possuem mais vínculos familiares. Para essas pessoas a alta assumirá outros significados, pois seu lar é a rua, os albergues, ou instituições de longa permanência. Essas situações de vulnerabilidade pressupõem uma abertura para ações intersetoriais e formação de redes de atenção que integrem a área da saúde com outras áreas relacionadas às necessidades do paciente ${ }^{(19)}$.

\section{Estratégias para continuidade do tratamento diante da alta hospitalar}

Esta categoria aponta as estratégias descritas pelos pacientes diante da alta hospitalar e a necessidade de continuidade ao tratamento. Os participantes trazem sua inserção em serviços extra-hospitalares, como o Centro de Atenção Psicossocial (CAPS), como uma possibilidade:

"Eu vou participar daquele grupo lá do CAPS. Daí,eles vão me acolher lá! Esse grupo é um grupo de gente que já tem problema mental, assim, que tem vontade de suicídio. Daí, a gente vai lá, faz atividade, tem médico. A gente consulta com os médicos de lá." (P3)

“[...] daí a gente vai para lá (para o CAPS) e faz atividade e passa o tempo e não fica só em casa." (P7)

De acordo com a Portaria n. ${ }^{\circ} 336$ de $2002^{(20)}$, o CAPS é definido como uma instituição de reabilitação social para pessoas que sofrem com algum transtorno mental, os quais devem oferecer um atendimento focado na reinserção psicossocial, em grupos, oficinas terapêuticas, visitas domiciliares, atendimento à família e atividades comunitárias ${ }^{(21)}$. Observa-se que a alta é vista como uma oportunidade de continuar o tratamento, não só medicamentoso, mas também o fato de participar de atividades terapêuticas de reinserção social(21)

A reabilitação psicossocial deve ser pautada nas três esferas da vida em sociedade: a casa, o trabalho e o lazer. Essa reinserção deve ocorrer com o resgate da autonomia, exercendo o papel de cidadão das pessoas com 
transtorno mental, realizando novas relações sociais. O CAPS desenvolve a função de compreender a pessoa como um ser singular, não mantendo o foco somente na doença ${ }^{(22)}$.

Para isso, o CAPS dispõe de práticas terapêuticas que são pautadas em um trabalho coletivo, realizando intervenções multidisciplinares e que envolvem a participação da família e do indivíduo. Dentre as atividades, estão: acolhimento; grupos e oficinas terapêuticas; visitas domiciliares; assembleias; atendimento com psiquiatra, psicólogo, assistente social e terapeutas ocupacionais. Essas práticas englobam as atividades individuais, como psicoterapias, manejos e o uso de psicofármacos, mas, além disso, abrangem o sujeito enquanto ser social sobre um aspecto ampliado, incluindo a atenção à família e inserindo atividades de lazer que contribuem para a retomada de sua independência, reafirmando a segurança nos ambientes em que transita ${ }^{(23)}$.

Um estudo evidencia, no entanto, que apesar dos usuários de saúde mental conhecerem o CAPS, há dificuldade em se adaptarem e se vincularem a esse serviço. Observando as reinternações, é necessário refletir sobre o tratamento ofertado nos CAPS, que pode não corresponder às expectativas e necessidades individuais dos usuários ${ }^{(24)}$.

O Projeto Terapêutico Singular (PTS) surge como uma estratégia interdisciplinar e multiprofissional necessária e eficaz durante a internação e após a alta hospitalar para identificar as necessidades das pessoas de acordo com seu grupo familiar e território em que está inserido ${ }^{(25)}$. Com ele é possível resgatar a história familiar, aspectos clínicos do usuário e traçar estratégias juntamente com os envolvidos, para que, no momento da alta, já exista o contato com a equipe de referência, o CAPS ou Unidade de Atenção Básica, o que facilita na adesão e continuidade do tratamento ${ }^{(26)}$.

A Atenção Básica configura-se como um ponto da Rede de Atenção da Saúde (RAS) que pode implementar novas práticas de cuidado em saúde mental, sendo de grande valia para o usuário que pode ter a continuidade do cuidado e do tratamento próximo da realidade onde vive ${ }^{(27)}$. Assim, é possível perceber o quão importante e necessário é investir em estratégias de reabilitação e cuidado de saúde mental no território, centrando o cuidado no usuário e no seu contexto familiar, e não somente na doença; desenvolvendo atividades que promovam a prevenção e promoção em saúde mental, efetivando na prática cotidiana o conceito ampliado do processo de saúde-doença ${ }^{(27)}$.

Aliado a isso, um estudo evidencia que a falta de acompanhamento nas redes de atenção à saúde após a internação hospitalar e a falta de instrumentos que viabilizem um plano de cuidados longitudinal e integrado podem ser determinantes nas possíveis reinternações e adesão ao tratamento ${ }^{(28)}$. Ademais, o hospital deve utilizar instrumentos que viabilizem a articulação dos diversos níveis de atenção, pois possibilitam a corresponsabilização do cuidado e também a garantia do encaminhamento ao serviço de referência para que o usuário receba a assistência de saúde de que necessita ${ }^{(28)}$.

Outros participantes do presente estudo descrevem como estratégias para dar continuidade ao tratamento após a alta hospitalar a necessidade de melhorar o autocuidado e a adesão aos medicamentos:

"Eu acho assim, que deu alta do hospital, tu tem que te ajudar, e o quanto tu estava te ajudando aqui, tu tem que continuar se ajudando lá fora, porque não adianta tu ir lá pra fora e, daqui três ou quatro dias, está voltando aqui de novo. Continuar o tratamento dos remédios, continuar na psicoterapeuta..." (P8)

"Tomar os remédios direito, para não ter que voltar de novo." (P12)

As expressões demonstram a importância de continuar o tratamento medicamentoso para evitar uma próxima reinternação. Há o entendimento acerca da importância do uso correto dos fármacos, de realizar o acompanhamento com o psiquiatra, de aceitar a medicação como método de controle de alguma condição mental. A abordagem terapêutica psicossocial é de extrema importância para ajudar os pacientes a trabalhar as repercussões da doença em suas vidas, na compreensão do significado de estar doente, como também na conscientização sobre a necessidade de manter o tratamento medicamentoso ${ }^{(29)}$.

Apesar do grande número de medicações psiquiátricas existentes, nota-se que somente o uso dos medicamentos não garante que se elimine possibilidade de novas internações. Contudo a adesão à terapia medicamentosa deve ser observada, uma vez que, após a melhora de alguns sintomas, os usuários tendem a abandonar ou diminuir o uso ${ }^{(30,31)}$. Um fator que pode estar associado à baixa adesão são os efeitos colaterais que algumas medicações podem causar, além da sensação de "cura".

Outro estudo também evidenciou tal relação, demonstrando que o conhecimento do paciente acerca dos efeitos colaterais pode acarretar na baixa adesão aos medicamentos ${ }^{(32)}$. Essa concepção por parte dos usuários pode dificultar nas informações fornecidas pelos profissionais, por receio que os usuários acabem por não realizar o uso do medicamento, o que pode acarretar em danos a terapêutica do usuário.

O baixo nível de adesão aos psicofármacos também pode estar associado ao pouco conhecimento dos cuidadores e dos próprios usuários ${ }^{(33)}$. Assim, reforça-se a necessidade de ações educativas, tanto da família como do usuário, 
durante a internação, na preparação para alta e nos serviços que o acompanharão após a alta hospitalar, com informações acerca do uso e dos efeitos dos medicamentos, promovendo autonomia ao usuário, reordenando suas capacidades psicossociais e suas potencialidades no curso da sua vida ${ }^{(30)}$.

Diante disso, vale ressaltar a importância da internação para o ajuste terapêutico ou manejo de crise, também como um ambiente de preparação para a alta, e de articulações que garantam a integralidade e continuidade do cuidado aos usuários ${ }^{(34)}$. Nessa perspectiva, a alta hospitalar deve ser planejada e articulada, com os dispositivos da rede e, principalmente, com o usuário, respeitando os desejos e a autonomia do mesmo. Para isso, é necessária a criação de vínculo, parceria e apoio mútuo entre os profissionais de saúde nos diversos pontos da rede de atenção à saúde, pautada no diálogo, na troca de informações, para que se efetive a continuidade do cuidado e se construa uma rede mais sólida e resolutiva ${ }^{(35)}$.

A preocupação com a disseminação da informação e a educação em saúde, fundamentais para a tomada de decisão e elementos importantes da promoção de saúde, parece estar ligada ao empoderamento, percebido como procedimento de capacitação dos indivíduos e comunidades para adquirirem maior controle sobre os fatores pessoais, socioeconômicos e ambientais que atingem a saúde ${ }^{(36)}$.

Este estudo possui limitações intrínsecas a estudos qualitativos, como ter tido como cenário somente uma unidade de internação da área de saúde mental. Entretanto oferece a possibilidade de construção do conhecimento no âmbito do cuidado para alta hospitalar das pessoas em sofrimento psíquico internadas em âmbito hospitalar.

\section{CONSIDERAÇÕES FINAIS}

Com este estudo, compreendeu-se a percepção da pessoa em sofrimento psíquico internada em uma unidade de atenção psicossocial sobre a alta hospitalar. As mesmas percebem a alta de diversas formas através de sentimentos, expectativas e desejos. Foram revelados sentimentos expressos pelas pessoas que passam por um período de internação, como medo, sensação de conquista e de liberdade, também sentidas como algo natural.

Ressalta-se que os entrevistados perceberam a necessidade de continuar o tratamento, por meio de atividades vinculadas ao CAPS e em espaços informais, como através de terapias complementares e medicamentosas. A alta hospitalar é vista como uma nova fase na vida, como um recomeço junto à família e à sociedade, bem como uma conquista pessoal. Após a alta hospitalar, os pacientes com transtorno mental passam pelo desafio de buscar alternativas para ultrapassar os obstáculos ainda existentes.

O presente estudo contribui para que os profissionais, especialmente da saúde mental, reflitam acerca de suas ações e das orientações passadas às pessoas diante da alta hospitalar. A pesquisa poderá auxiliar na reorganização do processo de trabalho e na preparação da alta hospitalar, assim como no aperfeiçoamento na atenção à saúde dos usuários internados.

Além disso, evidencia-se o quão imprescindível é que se invista no uso de instrumentos e estratégias, como o Projeto Terapêutico Singular e a alta assistida, para fomentar a vinculação dos usuários nos serviços da rede de atenção à saúde e, dessa forma, efetivar o cuidado longitudinal. Considerando os benefícios que esses instrumentos podem trazer, ressalta-se que eles devem ser utilizados em todas as áreas da saúde e em todos os níveis de atenção.

\section{CONFLITOS DE INTERESSE}

Não houve conflitos de interesses na execução desta pesquisa.

\section{CONTRIBUIÇÕES}

Valentine Cogo Mendes contribuiu com a elaboração e delineamento do estudo; com a análise e interpretação dos dados; e com a redação e revisão do manuscrito. Marlene Gomes Terra, Zaira Letícia Tisott, Daiana Foggiato Siqueira e Bruno Vinicius Rodrigues contribuíram com a análise e interpretação dos dados. Valquíria Toledo Souto contribuiu com a análise e interpretação dos dados; e com a redação e revisão do manuscrito.

\section{REFERÊNCIAS}

1. Focault M. História da Locura. 9a ed. São Paulo: Perspectiva; 2010.

2. Ministério da Saúde (BR). Lei N 10.216, de 6 de abril de 2001. Dispõe sobre a proteção e os direitos das 
pessoas portadoras de transtornos mentais e redireciona o modelo assistencial em saúde mental. Brasília: MS; 2001.

3. Pessoa JJM, Santos RCA, Clementino KKDO, Miranda FAN. A política de saúde mental no contexto do hospital psiquiátrico: desafios e perspectivas. Esc Anna Nery. 2016;20(1):83-9.

4. Alves PIC, Ferreira LA, Cardoso RJ. The Performance of the Family Health Stratgy Nurse in The Care of clientes in Psychic Suffering. Texto \& Contexto Enferm. 2015;20(1):85-93.

5. Flesch LD, Araujo TCCF. Alta hospitalar de pacientes idosos: Necessidades e desafios do cuidado contínuo. Estud Psicol (Natal). 2014;19(3):157-238.

6. Delatorre PG, Sá SPC, Valente GSC, Silvino ZR. Planejamento para a alta hospitalar como estratégia de cuidado de enfermagem: revisão integrativa. Rev Enferm UFPE. 2013;7(esp):7151-9.

7. Ministério da Saúde (BR). Política Nacional de Promoção da Saúde. Brasília: MS; 2006.

8. Minayo MCS. O desafio do conhecimento: pesquisa qualitativa em saúde. 14a ed. São Paulo: Hucitec; 2014.

9. Turato ER. Tratado da metodologia da pesquisa clínico-qualitativa: construção teórico-epistemológica, discussão comparada e aplicação nas áreas da saúde e humanas. 5ª ed. Petrópolis: Vozes; 2011.

10. Nobre FC, Corrêa DA, Nepomuceno LH, Souza AJ, Siqueira VF. A amostragem na Pesquisa de natureza científica em um campo multiparadigmático: peculiaridades do método qualitativo. Revista Espacios. 2017;38(22):13.

11. Conselho Nacional de Saúde (BR). Resolução No 466, 12 de dezembro de 2012. Dispõe sobre as diretrizes e as normas regulamentadoras de pesquisa envolvendo seres humanos. Brasília: Conselho Nacional de Saúde; 2012.

12. Ely GZ, Terra MG, Silva AA, Freitas FF, Padoin SM, Lara MP. Internação psiquiátrica: significados para usuários de um centro de atenção psicossocial. SMAD, Rev Eletrônica Saúde Mental Álcool Drog. 2014;10(1):23-28.

13. Ely GZ, Terra MG, Silva AA, Freitas FF, Leite MT, Brum BN. Percepções do ser humano internado em unidade psiquiátrica sobre o viver com doença mental. Texto \& Contexto Enferm. 2017;26(3):e0280016.

14. Furlan MM. O sentido de ser internado em hospital psiquiátrico à luz da fenomenologia de Heidegger [tese]. Ribeirão Preto: USP; 2008.

15. Castro AS, Furegato ARF, Santos JLF. Egressos de internação psiquiátrica acompanhados na rede de serviços de saúde. Rev Enferm Atenção Saúde. 2018;7(1):152-65.

16. Martins PPS, Guanaes-Lorenzi C. Participação da família no tratamento em saúde mental como prática no cotidiano do serviço. Psicol Teor Pesq. 2016;32(4):1-9.

17. Souto VT, Terra MG, Soccol KLS, Mostardeiro SCTS, Xavier MS, Teixeira JK. Cuidado da equipe de enfermagem na percepção de familiares de pacientes psiquiátricos. Rev Enferm UFPE. 2015;9(2):910-17.

18. Duarte MLC, Carvalho J, Brentano V. Percepção dos familiares acerca do grupo de apoio realizado em uma unidade de internação psiquiátrica. Rev Gaúch Enferm. 2018;39:e2017-0115.

19. Gama CAP, Campos RTO, Ferrer AL. Saúde mental e vulnerabilidade social: a direção do tratamento. Rev Latinoam Psicopatol Fundam. 2014;17(1):69-84.

20. Ministério da Saúde (BR). Portaria GM n. 336, de 19 de fevereiro de 2002. Define e estabelece diretrizes para o funcionamento dos Centros de Atenção Psicossocial. Brasília: MS; 2002.

21. Weber CAT, Juruena MF. Inclusão de usuários de hospital-dia em saúde mental: uma revisão. Psicol Saúde Doenças. 2014;15(3):790-9.

22. Pacheco SUC, Rodrigues SR, Benatto MC. A importância do empoderamento do usuário de CAPS para a (re)construção do seu projeto de vida. Mental. 2018;12(22):72-89.

23. Franzmann UT, Kantorski LP, Jardim VMR, Treichel CAS, Oliveira MM, Pavani FM. Fatores associados à percepção de melhora por usuários de Centros de Atenção Psicossocial do Sul do Brasil. Cad Saúde Pública. 2017;33(7):00085216 
24. Pereira AR, Joazeire G. Percepção da internação em hospital psiquiátrico por pacientes com diagnóstico de esquizofrenia. Mundo Saúde. 2015;39(4):476-83.

25. Miranda FAC, Coelho EBS, Moré CLOO. Projeto terapêutico singular Universidade Federal de Santa Catarina. Florianópolis: Universidade Federal de Santa Catarina; 2012.

26. Ribeiro MC, Waldes CB, Ribeiro MC, Bezerra WC. A reabilitação psicossocial como estratégia de cuidado: percepções e práticas desenvolvidas por trabalhadores de um serviço de saúde mental. Rev Enferm UFPE. 2015;9(2):910-17.

27. Gonzales AM. Planos de Ações na Promoção e Prevenção de Transtornos em Saúde Mental na Atenção Primária à Saúde [monografia]. Minas Gerais: Universidade Federal de Minas Gerais; 2015.

28. Zanardo GLP, Silveira LHC, Rocha CMF, Rocha KB. Internações e reinternações psiquiátricas em um hospital geral de Porto Alegre: características sociodemográficas, clínicas e do uso da Rede de Atenção Psicossocial. Rev Bras Epidemiol. 2017;20(3):460-74.

29. Xavier MS, Terra MG, Silva CT, Mostardeiro SCTS, Silva AD, Freitas FF. O significado da utilização de psicofármacos para indivíduos com transtorno mental em acompanhamento ambulatorial. Esc Anna Nery. 2014;18(2)323-9.

30. Xavier MS, Terra MG, Silva CT, Souto VT, Mostardeiro SCTS, Vasconcelos RO. A utilização de psicofármacos em indivíduos com transtorno mental em acompanhamento ambulatorial. Enferm Glob. 2014;13(36):114-25.

31. Guedes AC, Olschowsky A, Kantorski LP, Antonacci MH. Transferência de cuidados: processo de alta dos usuários de um centro de atenção psicossocial. Rev Eletrônica Enferm. 2017;19:43794.

32. Brito ESV, Arena CA. Access to information on diagnosing and hospitalizing an individual with mental illness. SMAD, Rev Eletrônica Saúde Mental Alcool Drog. 2015;11(1):11-8.

33. Silva JC, Herzog LM. Psicofármacos e psicoterapia com idosos. 2015;27(2):438-48.

34. Maier ES, Mello AL, Siqueira DF, Terra MG. Instrumento para encaminhamento de usuários a serviços substitutivos de saúde mental: relato de experiência. Rev Soc Hum. 2017;30(3):205-12.

35. Zanardo GL, Bianchessi DLC, Rocha KB. Dispositivos e conexões da rede de atenção psicossocial (RAPS) de Porto Alegre - RS. Est Inter Psicol. 2018;9(3):80-101.

36. World Health Organization. Health promotion evaluation: recommendations to policymakers. Copenhagen: European Working Group on Health Promotion Evaluation; 1998.

\section{Autor para correspondência:}

Valentine Cogo Mendes

Secretaria da Residência Multiprofissional em Saúde - UFSM

Avenida Roraima, 1000/ Prédio 26 - $3^{\circ}$ Andar - Sala 1356

Bairro: Cidade Universitária - Camobi

CEP: 97105-900 - Santa Maria - RS - Brasil

E-mail: valentinecmendes@gmail.com

Como citar: Mendes VC, Tisott ZL, Souto VT, Terra MG, Siqueira DF, Rodrigues BV. Percepção sobre a alta hospitalar de pessoas internadas em unidade de atenção psicossocial. Rev Bras Promoç Saúde. 2019;32:9394. 